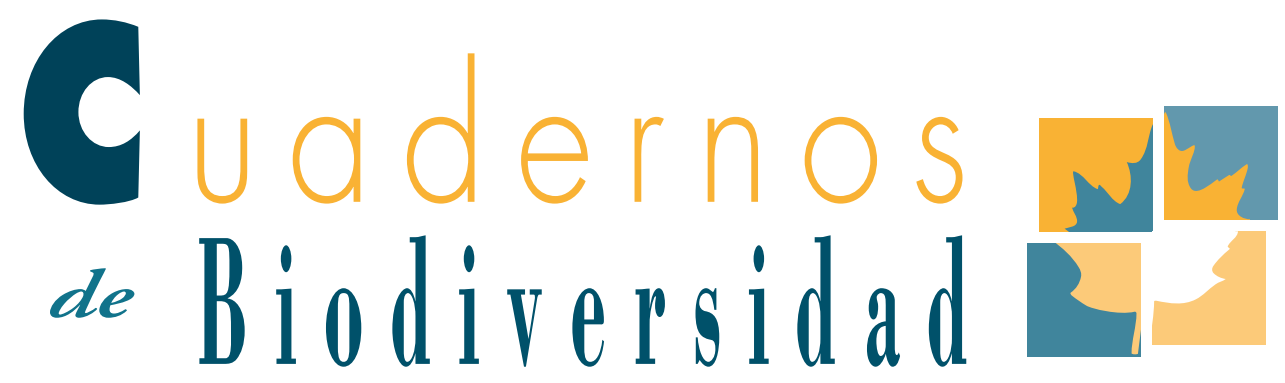

Cuadernos de Biodiversidad 62 (2022): 1-13

ISSN: 2254-6I $2 \mathrm{X}$

DOI:IO.I4I98/CDBIO.2022.62.0I

\title{
Los chopos cabeceros de La Yesa (Valencia): un paraíso de biodiversidad
}

The black poplar pollards of La Yesa (Valencia): a paradise of biodiversity

Roque BelengueR ${ }^{\mathrm{I}}$

${ }^{\text {I }}$ Consultor ambiental, España

roque.belenguer@gmail.com

Recibido: 13/09/2021

Aceptado: 22/12/2021

Publicado: 27/01/2022

(C) 2022 Roque Belenguer

LICENCIA:

Este trabajo se publica bajo una

Licencia Creative Commons

Reconocimiento 4.0 Internacional.

\section{(c) (i)}

\section{Cómo CITAR:}

Belenguer, R. (2022). Los chopos cabeceros de La Yesa (Valencia): un paraíso de biodiversidad. Cuadernos de Biodiversidad (62), 1-13. https://doi. org/10.14198/cdbio.2022.62.01

\section{RESUMEN}

El presente trabajo da a conocer la gran importancia de los chopos cabeceros ubicados en La Yesa (Valencia), unos árboles manejados durante generaciones por la población rural. Se destaca su alto valor paisajístico, biológico y cultural, así como la necesidad de gestionar este espacio natural para poder preservarlo para las generaciones venideras.

Pallabras clave: Chopos cabeceros; La Yesa; biodiversidad; servicios ecosistémicos; conservación; gestión.

\section{ABSTRACT}

The present work shows the great importance of the black poplar pollards located in La Yesa (Valencia), trees that have been managed for generations by the rural population. Its high landscape, biological and cultural value is highlighted, as well as the need to manage this natural space in order to preserve it for future generations.

Key words: Black poplar pollards; La Yesa; biodiversity; ecosystem services; conservation; management. 


\section{EL MUNICIPIO DE LA YESA}

En el interior de la provincia de Valencia se sitúa la comarca de La Serranía, una de las zonas más agrestes y desconocidas de la Comunitat Valenciana. La influencia climática y orográfica de la Sierra de Javalambre, estribación del Sistema Ibérico, se hace patente especialmente en el norte de este territorio. Justo a los pies de esta sierra, se encuentra el pueblo de La Yesa, a unos 1000 m s. n. m En su término municipal aparecen los termotipos meso y supramediterráneos y los ombrotipos seco y subhúmedo (Mateo et al., 2011).

Desde el punto de vista biogeográfico La Yesa pertenece a la provincia Mediterránea Ibérica Central, y dentro de esta a la subprovincia Oroibérica y al sector Ibérico Maestracense (Mateo et al., 2011). En las cotas más altas del municipio, a $1500 \mathrm{~m}$ s. n. m., aparecen interesantes sabinares rastreros de Juniperus sabina Linnaeus. A continuación, los pinares de Pinus sylvestris Linnaeus y Pinus nigra J.F. Arnold subsp. salzmannii (Dunal) Franco cobran importancia en las laderas de todas estas estribaciones de Javalambre. En las zonas más bajas y con menor pendiente, sobre los 1000-1200 m s. n. m., aparecen cultivos de secano -básicamente cereal, vid y almendro- y sabinares albares de Juniperus thurifera Linnaeus en zonas con suelo pobre y rocoso. En el área de estudio sobreviven también poblaciones de invertebrados típicos de estos hábitats montanos, en los que destaca la escasa mariposa apolo (Parnassius apollo Linnaeus) o la isabelina (Graellsia isabellae Graells), considerada por muchos entomólogos como la mariposa más bella de Europa.

\section{UNA CHOPERA AUTÓCTONA}

La mayoría de ramblas y barrancos de La Yesa están gran parte del año sin agua, por lo que los bosques ribereños son prácticamente inexistentes. No obstante, la rambla de Ahíllas mantiene un pequeño caudal a lo largo de casi todo el ciclo anual que alberga una interesante y singular chopera de Populus nigra Linnaeus. Estos chopos, también conocidos como álamo negro, pollancre o xop, son árboles caducifolios y dióicos que pueden alcanzar una altura de hasta
$30 \mathrm{~m}$ y son de crecimiento muy rápido. Se trata de ejemplares autóctonos, identificados por su porte y características morfológicas de las hojas, yemas y corteza, lo cual los diferencia de otras variedades o subespecies (López, 2001). Cuentan con una distribución muy restringida y localizada debido a la utilización extendida en muchas de las riberas de todo el territorio de la especie híbrida Populus $x$ canadensis Moench, procedente del cruce de P. nigra con la especie norteamericana $P$. deltoides Bartram ex Marshall. Estos híbridos han sido cultivados con fines ornamentales y para la obtención de pasta de papel y madera para cajas ligeras para fruta, habiendo sustituido a la mayoría de choperas ibéricas genéticamente puras (Figura 1).

Los 200 cabeceros censados en la rambla de Ahíllas ocupan un tramo de unos $2 \mathrm{~km}$ lineales, a una altura de $900 \mathrm{~m}$ s. n. m. También aparecen otros chopos cabeceros aislados en los alrededores del pueblo de La Yesa, algunos de ellos muertos tras haber sufrido una severa escamonda hace ya varias décadas. En la cercana aldea de La Canaleja de Arriba, perteneciente al municipio de Alpuente, se puede contemplar un individuo solitario de gran porte y catalogado como árbol monumental por la Generalitat Valenciana (RESOLUCIÓN de 15 de enero de 2020, del director general de Medio Natural y de Evaluación Ambiental, por la que se actualiza el Catálogo de Árboles Monumentales y Singulares de la Comunitat Valenciana).

Esta chopera valenciana es muy pequeña en comparación con otros núcleos del sur de Aragón, como la situada en la cuenca del río Pancrudo, con más de 20000 chopos cabeceros. Sin embargo, su verdadera importancia radica en su aislamiento y singularidad. Las principales choperas más cercanas de Teruel están situadas a más de $60 \mathrm{~km}$, en la cuenca del río Alfambra.

Se desconocen las razones por las cuales no existen otros ejemplos de chopos cabeceros en otras localidades cercanas, como el Rincón de Ademuz o la Sierra de Javalambre. Ello hace que esta chopera sea de gran interés biogeográfico y etnológico y, por lo tanto, debería de ser protegida. Por otro lado, habría que profundizar en el estudio de su biodiversidad asociada y realizar una caracterización ecológica en detalle. Todo ello con un claro objetivo: conocer para poder conservar. 


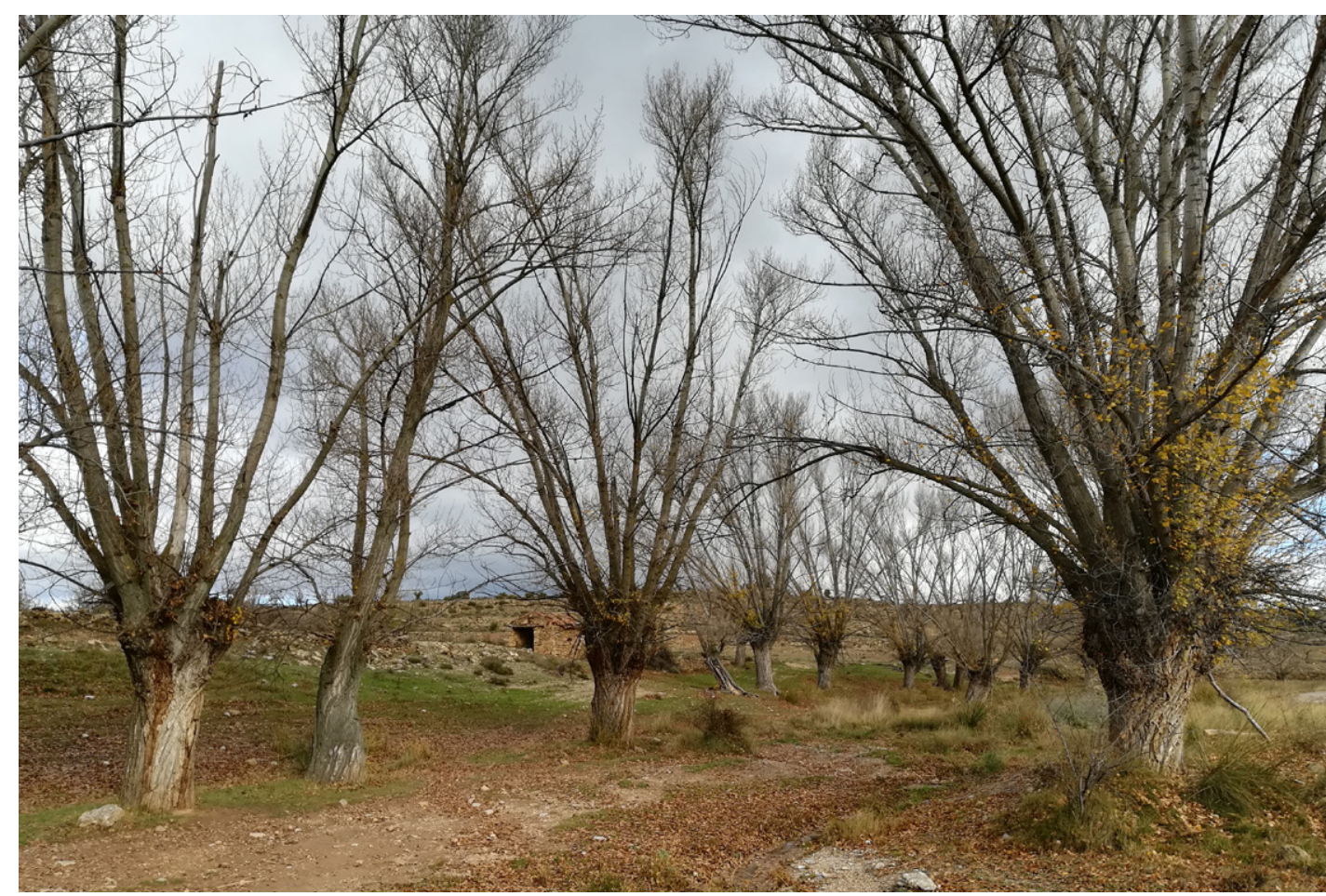

Figura 1. Los chopos cabeceros de La Yesa configuran un paisaje de alto valor estético, biológico y cultural.

\section{CARACTERISTICAS DE LOS CHOPOS CABECEROS}

Gran parte de los chopos de la rambla de Ahíllas son muy viejos, de gran porte y presentan una fisionomía muy particular. El tronco es relativamente corto, derecho y con un ensanchamiento a unos $2 \mathrm{~m}$ de altura, y las ramas parten de manera muy vertical desde esa "cabeza". Esta apariencia es producto de una técnica de poda, la escamonda, que consiste en la corta de las ramas principales de manera regular, en turnos de entre 12 y 15 años, que pueden llegar hasta los 20 años en los parajes más fríos. En el sur Aragón a este tipo de árboles se les llama "cabeceros". El ensanchamiento del tronco es debido a la reiteración del corte de las ramas en un mismo punto. Las ramas más gruesas eran utilizadas básicamente como vigas para la construcción. Las ramas más finas y las hojas eran utilizadas como suplemento alimenticio para el ganado (de Jaime y Herrero, 2007). En menor medida, la madera también era aprovechada como leña para los hogares, debido a que eran una de las pocas fuentes de madera en una zona donde se ha dado una intensa deforestación (Figura 2).

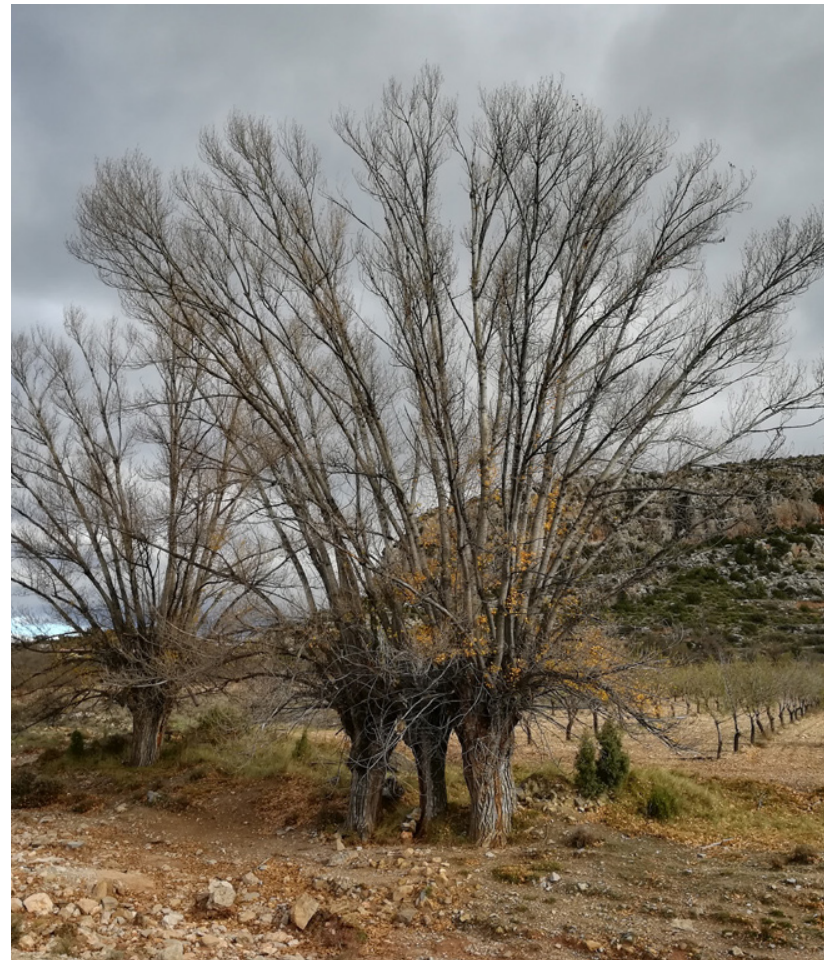

Figura 2. La rambla de Ahíllas está situada en un altiplano muy deforestado del Sistema Ibérico. 


\section{UNA BIODIVERSIDAD DESBORDANTE}

Con el paso de los años los chopos cabeceros van aumentando el perímetro del tronco, con el consecuente incremento de huecos, grietas y corteza muerta. En ese proceso, estos añejos organismos se convierten en un rico y biodiverso ecosistema, pues la multitud de hendiduras, agujeros, charcas, rezumados y superficies varias da lugar a hábitats y nichos en los que puede vivir un nutrido número de especies, como hongos, líquenes, musgos, algas, invertebrados, vertebrados, etc. (de Jaime y Herreros, 2007). Como ejemplo, en los chopos cabeceros de Aragón, durante un trabajo todavía no finalizado, se han identificado por el momento más de 150 especies de coleópteros saproxílicos (Marcos Méndez, com. pers.), que utilizan durante parte de su ciclo vital la madera muerta, hongos xilófagos u otros organismos asociados a la madera (Méndez, 2009) (Figura 3).

Además, en Teruel han sido registradas 15 especies de murciélagos asociadas a los chopos cabeceros (Lorente, 2014).
En la chopera de la rambla de Ahíllas se dispone de información propia sobre las aves, con 131 especies pertenecientes a 34 familias (Tabla 1), y los mamíferos, con 30 especies correspondientes a 16 familias (Tabla 2). Destacan los quirópteros, de los que se han encontrado nueve especies, en un inventario que se está llevando a cabo en la actualidad, basado en grabaciones de ultrasonidos y su posterior identificación, en colaboración con la Brigada de Fauna del Servicio de Vida Silvestre de la Generalitat Valenciana. Cabe incidir en la presencia de especies forestales, como el murciélago de bosque (Barbastella barbastellus Schreber), muy dependiente de bosques maduros y de la existencia de grietas y huecos en la corteza de árboles viejos (Gutiérrez et al., 2021). Sería recomendable realizar más muestreos y trampeos para confirmar la presencia en La Yesa de otras especies de murciélagos arborícolas, como el orejudo dorado (Plecotus auritus Linnaeus), el nóctulo menor (Nyctalus leisleri Kuhl) o el murciélago ratonero forestal (Myotis bechsteinii Kuhl).

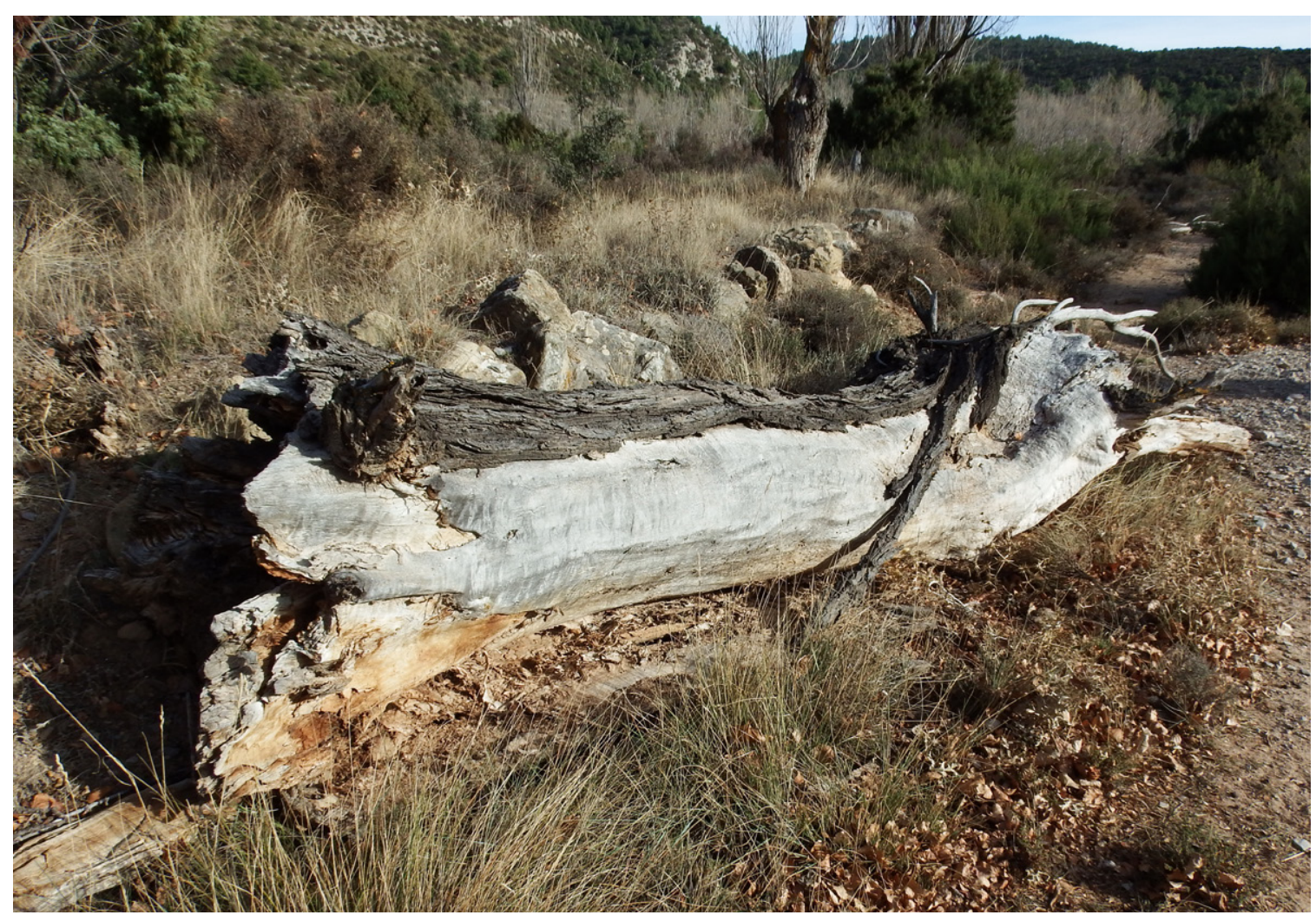

Figura 3. La disponibilidad de madera muerta es fundamental para el correcto funcionamiento del ecosistema ribereño. 
Parte de esta ribera ha sido aprovechada secularmente por el ganado ovino, lo cual ha generado espacios arbóreos abiertos o adehesados, con un estrato bajo constituido por herbáceas y junqueras, un hábitat de interés comunitario en el cual aparecen poblaciones de topillo de Cabrera (Microtus cabrerae Thomas) y rata de agua (Arvicola sapidus Miller). Ambas especies son muy escasas y están amenazadas, tanto a nivel autonómico como nacional y europeo (Figura 4).

Además de albergar una elevada biodiversidad el chopo negro es una especie pionera en los procesos de sucesión de los bosques de ribera y juega un importantísimo papel contribuyendo con funciones ecosistémicas relacionadas con la regulación térmica en estos ambientes riparios. A través de su sistema radicular y su follaje denso, absorbe y transpira grandes cantidades de agua, aumentando la humedad relativa del entorno. A su vez, sus raíces son un excelente protector de los márgenes y sus hojas muertas ayudan a aumentar la fertilidad del suelo y reducir la erosión superficial (de Jaime y Herrero, 2007).

\section{AMENAZAS Y OPCIONES DE GESTIÓN}

La presencia de estos fabulosos árboles, manejados durante generaciones por la población rural, ha modelado un paisaje $y$, por ende, un patrimonio que hay que conservar. Desafortunadamente, se enfrentan a un conjunto de amenazas.

Los chopos cabeceros de Aragón han sido muy bien estudiados desde hace ya bastantes años. Las principales amenazas e impactos detectados derivan del imparable éxodo rural acaecido en las zonas de interior desde mediados del siglo XX. El abandono de la escamonda y otros usos relacionados con estos chopos tienen como consecuencia la caída de ramas, desgaje de troncos y la posterior muerte del árbol. A su vez, el descenso del nivel freático, debido a la disminución de las precipitaciones y la mayor frecuencia de sequías por causa del cambio climático, impiden el correcto desarrollo de la vegetación ribereña por estrés hídrico. Tampoco hay que olvidar el abuso en la explotación de las aguas subterráneas, con similares consecuencias (de Jaime y Herrero, 2007) (Figura 5).

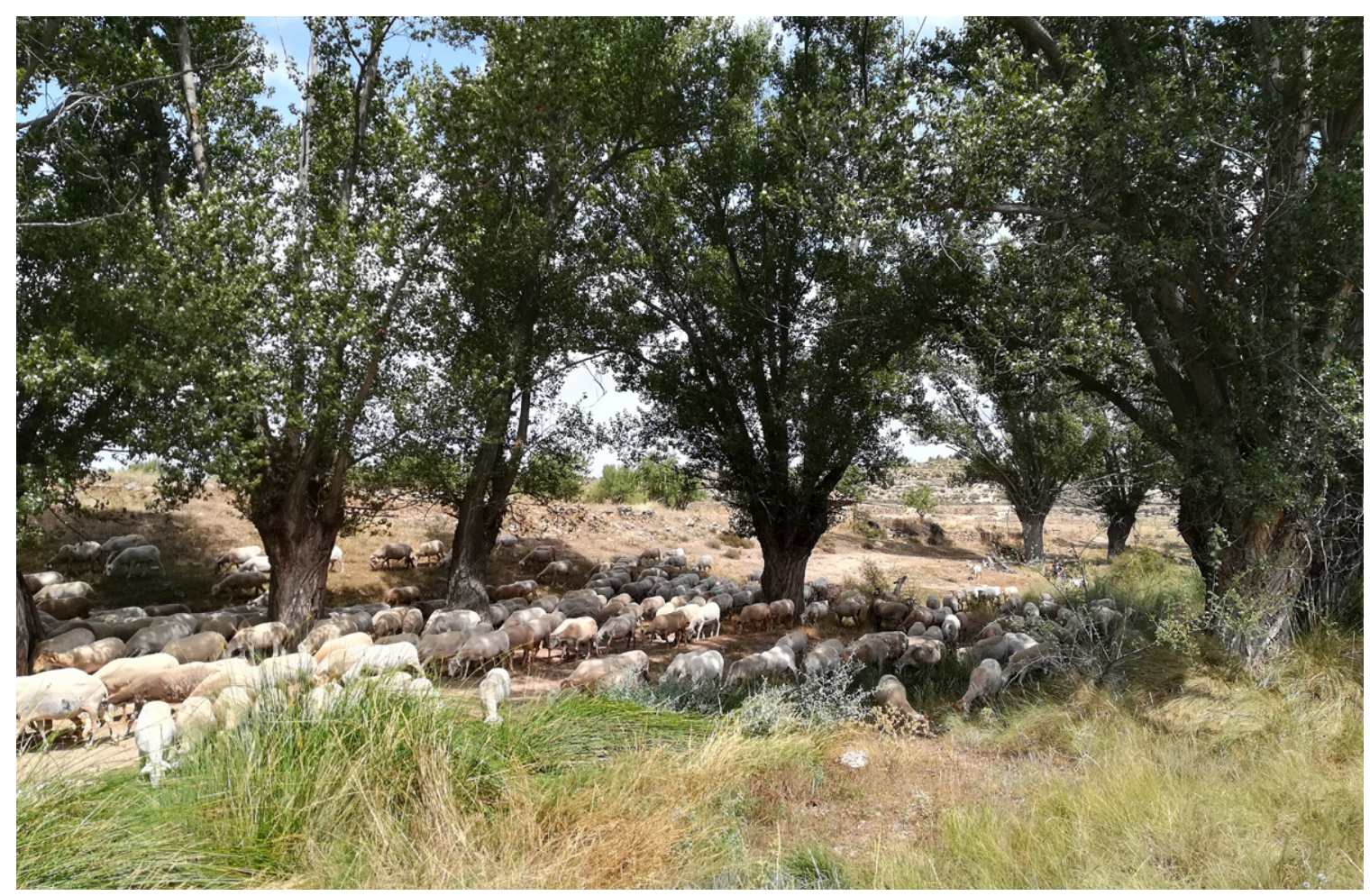

Figura 4. Con los años se ha ido perdiendo la mayoría de los usos y costumbres que motivaron la plantación de estos árboles, pero su utilización por parte del ganado ovino todavía perdura en este bello paraje. 


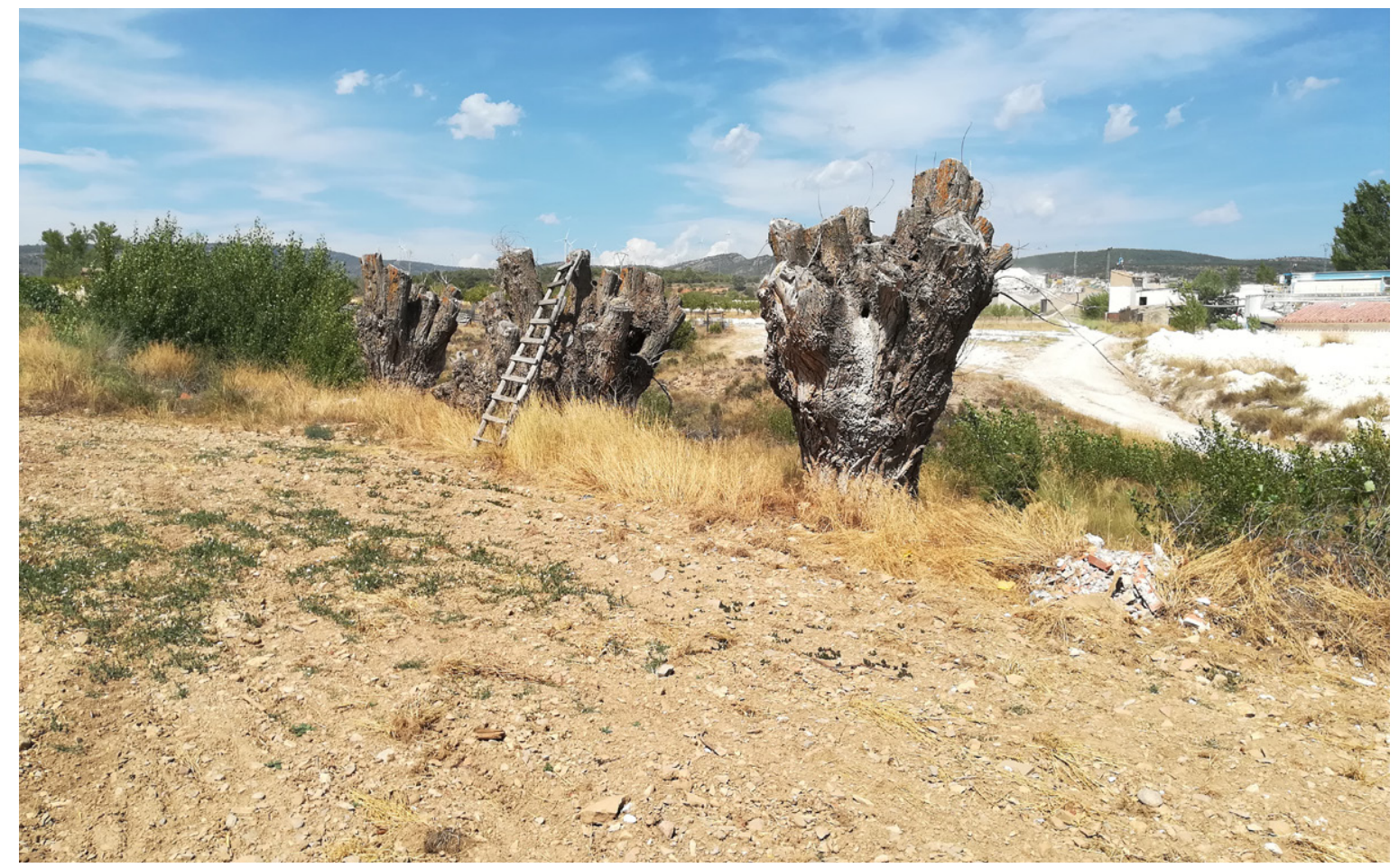

Figura 5. La desaparición de estos chopos está estrechamente relacionada con el éxodo rural y el abandono de sus aprovechamientos tradicionales.

Otras actuaciones que pueden ocasionar graves daños a estas formaciones arbóreas son las limpiezas de cauce ejecutadas por las confederaciones hidrográficas, con la eliminación de árboles muertos y ramas. Son totalmente incompatibles con la preservación de los ecosistemas ribereños, por lo que habría que evitarlas en la medida de lo posible.

Entre las medidas barajadas para gestionar y conservar la chopera de la rambla de Ahíllas, la Arboleda Singular de Interés Local es la más fácilmente abordable en un primer paso. El Decreto 154/2018 del Consell desarrolla la Ley 4/2006 de la Generalitat, de Patrimonio Arbóreo Monumental de la Comunitat Valenciana. Su principal objetivo es regular la protección, conservación, difusión, fomento, investigación y acrecentamiento del patrimonio arbóreo monumental valenciano. Este decreto establece que se podrá declarar con la categoría de arboleda singular aquellos conjuntos arbóreos que, sin llegar a alcanzar la categoría de monumental, destaquen por sus características notables de edad, porte u otros tipos de acontecimientos históricos, culturales, científicos, de recreo o ambientales que los hagan merecedores de medidas de protección y conservación específica. Obviamente, la chopera de La Yesa cumple con todos los requisitos para esta declaración.

La declaración como Paraje Natural Municipal es más engorrosa, lenta y precisa de mucha burocracia, lo cual no la hace muy adecuada a corto-medio plazo. Sin embargo, son más factibles las figuras de Reserva de Fauna Silvestre, por la presencia de topillo de Cabrera, especie catalogada como Vulnerable por la normativa autonómica, o la figura de Microrreserva de Flora por la presencia de plantas de interés asociadas a la chopera, como por ejemplo Veronica beccabunga Linnaeus y orquídeas típicas de enclaves frescos de los géneros Epipactis y Dactylorhiza, así como otras especies que puedan ser detectadas en futuras prospecciones.

Como precedente de estas opciones de gestión, en Teruel se creó el Parque Cultural del Chopo Cabecero del Alto Alfambra en 2018, todo un referente europeo en lo que respecta a la gestión y estudio de los árboles trasmochos (parquechopocabecero.com). La figura del chopo cabecero se posiciona como un importante generador de cultura y sabiduría popular. A su vez, en el río Alfambra el mismo año se 
declaró una Arboleda Singular denominada "Ribera de Chopo Cabecero". El tramo supera los $12 \mathrm{~km}$ de longitud y una superficie de más de 50 ha. Sin duda, Aragón es un claro modelo a seguir e imitar.

Los chopos cabeceros de la rambla de Ahíllas componen un paisaje irrepetible, conforman un valioso patrimonio etnológico y realizan funciones ecológicas trascendentales en el medio ribereño. Además, su material genético intacto es de vital interés para la supervivencia de Populus nigra, dada la excesiva proliferación de plantaciones de híbridos por toda la geografía ibérica. Es nuestra obligación salvaguardar esta formación única e irrepetible y lograr en un futuro no muy lejano su conservación y protección.

\section{AGRADECIMIENTOS}

Todo lo que he aprendido sobre el universo del chopo cabecero se lo debo a Chabier de Jaime Lorén, Director-Gerente del Parque Cultural del Chopo Cabecero del Alto Alfambra, un gran entusiasta y excelente divulgador de este patrimonio arbóreo.

Tabla 1: Relación de especies de aves registradas en la zona de estudio.

\begin{tabular}{|c|c|c|}
\hline NOMBRE CIENTÍFICO & NOMBRE VALENCIANO & NOMBRE CASTELLANO \\
\hline \multicolumn{3}{|l|}{ Fam. Phasianidae } \\
\hline Alectoris rufa & Perdiu comuna & Perdiz roja \\
\hline Coturnix coturnix & Guatla & Codorniz común \\
\hline \multicolumn{3}{|l|}{ Fam. Ardeidae } \\
\hline Ardea cinerea & Agró blau & Garza real \\
\hline \multicolumn{3}{|l|}{ Fam. Accipitridae } \\
\hline Pernis apivorus & Pilot & Abejero europeo \\
\hline Milvus migrans & Milà negre & Milano negro \\
\hline Milvus milvus & Milà reial & Milano real \\
\hline Neophron percnopterus & Miloca & Alimoche común \\
\hline Gyps fulvus & Voltor comú & Buitre leonado \\
\hline Circaetus gallicus & Àguila serpera & Culebrera europea \\
\hline Circus aeruginosus & Arpellot de marjal & Aguilucho lagunero occidental \\
\hline Circus cyaneus & Arpellot pàl.lid & Aguilucho pálido \\
\hline Accipiter gentilis & Astor & Azor común \\
\hline Accipiter nisus & Esparver & Gavilán común \\
\hline Buteo buteo & Aguilot comú & Busardo ratonero \\
\hline Aquila chrysaetos & Àguila reial & Águila real \\
\hline Aquila pennata & Àguila calçada & Águila calzada \\
\hline Aquila fasciata & Àguila de panxa blanca & Águila perdicera \\
\hline \multicolumn{3}{|l|}{ Fam. Pandionidae } \\
\hline Pandion haliaetus & Àguila pescadora & Águila pescadora \\
\hline
\end{tabular}




\begin{tabular}{|c|c|c|}
\hline Fam. Falconidae & & \\
\hline Falco tinnunculus & Xoriguer comú & Cernícalo vulgar \\
\hline Falco columbarius & Esmerla & Esmerejón \\
\hline Falco subbuteo & Falconet & Alcotán europeo \\
\hline Falco peregrinus & Falcó pelegrí & Halcón peregrino \\
\hline \multicolumn{3}{|l|}{ Fam. Columbidae } \\
\hline Columba livia & Colom roquer & Paloma bravía \\
\hline Columba oenas & Sisella & Paloma zurita \\
\hline Columba palumbus & Todó & Paloma torcaz \\
\hline Streptopelia turtur & Tórtora & Tórtola europea \\
\hline \multicolumn{3}{|l|}{ Fam. Cuculidae } \\
\hline Cuculus canorus & Cuquello & Cuco común \\
\hline \multicolumn{3}{|l|}{ Fam. Strigidae } \\
\hline Otus scops & Corneta & Autillo europeo \\
\hline Bubo bubo & Brúfol & Búho real \\
\hline Athene noctua & Mussol & Mochuelo europeo \\
\hline Strix aluco & Caro & Cárabo común \\
\hline \multicolumn{3}{|l|}{ Fam. Caprimulgidae } \\
\hline Caprimulgus europaeus & Enganyapastors & Chotacabras europeo \\
\hline Caprimulgus ruficollis & Saboc & Chotacabras cuellirrojo \\
\hline \multicolumn{3}{|l|}{ Fam. Apodidae } \\
\hline Apus apus & Falcia & Vencejo común \\
\hline Apus pallidus & Falcia pàl.lida & Vencejo pálido \\
\hline Apus melba & Falcia de panxa blanca & Vencejo real \\
\hline \multicolumn{3}{|l|}{ Fam. Alcedinidae } \\
\hline Alcedo atthis & Blavet & Martín pescador común \\
\hline \multicolumn{3}{|l|}{ Fam. Meropidae } \\
\hline Merops apiaster & Abellerol & Abejaruco europeo \\
\hline \multicolumn{3}{|l|}{ Fam. Coraciidae } \\
\hline Coracias garrulus & Cavallet & Carraca europea \\
\hline \multicolumn{3}{|l|}{ Fam. Upupidae } \\
\hline Upupa epops & Puput & Abubilla \\
\hline \multicolumn{3}{|l|}{ Fam. Picidae } \\
\hline Jynx torquilla & Formiguer & Torcecuello euroasiático \\
\hline Picus sharpei & Picacarrasques & Pito real ibérico \\
\hline Dendrocopos major & Picot garser gros & Pico picapinos \\
\hline
\end{tabular}




\begin{tabular}{|c|c|c|}
\hline Fam. Alaudidae & & \\
\hline Calandrella brachydactyla & Terrerolí & Terrera común \\
\hline Galerida cristata & Cogullada del topet & Cogujada común \\
\hline Galerida theklae & Cogullada de serra & Cogujada montesina \\
\hline Lullula arborea & Cotoliu & Alondra totovía \\
\hline Alauda arvensis & Terrerola & Alondra común \\
\hline \multicolumn{3}{|l|}{ Fam. Hirundinidae } \\
\hline Riparia riparia & Parpalló & Avión zapador \\
\hline Ptyonoprogne rupestris & Roquer & Avión roquero \\
\hline Hirundo rustica & Oroneta & Golondrina común \\
\hline Hirundo daurica & Oroneta cua-rogenca & Golondrina dáurica \\
\hline Delichon urbicum & Oronell & Avión común \\
\hline \multicolumn{3}{|l|}{ Fam. Motacillidae } \\
\hline Anthus campestris & Titeta d'estiu & Bisbita campestre \\
\hline Anthus trivialis & Titeta dels arbres & Bisbita arbóreo \\
\hline Anthus pratensis & Titeta d'hivern & Bisbita pratense \\
\hline Anthus spinoletta & Titeta de muntanya & Bisbita alpino \\
\hline Motacilla cinerea & Sequiolet & Lavandera cascadeńa \\
\hline Motacilla alba & Cueta blanca & Lavandera blanca \\
\hline \multicolumn{3}{|l|}{ Fam. Troglodytidae } \\
\hline Troglodytes troglodytes & Caragolet & Chochín común \\
\hline \multicolumn{3}{|l|}{ Fam. Prunellidae } \\
\hline Prunella modularis & Pardal de bardissa & Acentor común \\
\hline Prunella collaris & Cercavores & Acentor alpino \\
\hline \multicolumn{3}{|l|}{ Fam. Turdidae } \\
\hline Erithacus rubecula & Pit-roig & Petirrojo europeo \\
\hline Luscinia megarhynchos & Rossinyol & Ruiseñor común \\
\hline Phoenicurus ochruros & Fumat & Colirrojo tizón \\
\hline Phoenicurus phoenicurus & Cua-roja reial & Colirrojo real \\
\hline Saxicola rubetra & Cagamànecs rogenc & Tarabilla norteńa \\
\hline Saxicola rubicola & Cagamànecs & Tarabilla europea \\
\hline Oenanthe oenanthe & Còlbit gris & Collalba gris \\
\hline Oenanthe hispanica & Còlbit ros & Collalba rubia \\
\hline Oenanthe leucura & Còlbit negre & Collalba negra \\
\hline Monticola saxatilis & Merla roquera & Roquero rojo \\
\hline Monticola solitarius & Solitari & Roquero solitario \\
\hline Turdus torquatus & Merla de pit blanc & Mirlo capiblanco \\
\hline
\end{tabular}




\begin{tabular}{|c|c|c|}
\hline Turdus merula & Merla & Mirlo común \\
\hline Turdus pilaris & Tordanxa & Zorzal real \\
\hline Turdus philomelos & Tord comú & Zorzal común \\
\hline Turdus iliacus & Tord castellà & Zorzal alirrojo \\
\hline Turdus viscivorus & Griva & Zorzal charlo \\
\hline \multicolumn{3}{|l|}{ Fam. Sylvidae } \\
\hline Cettia cetti & Rossinyol bord & Cetia ruiseñor \\
\hline Cisticola juncidis & Estaranyit & Cistícola buitrón \\
\hline Sylvia undata & Busquereta cuallarga & Curruca rabilarga \\
\hline Sylvia conspicillata & Busquereta trencamates & Curruca tomillera \\
\hline Sylvia cantillans & Busquereta de garriga & Curruca carrasqueña \\
\hline Sylvia melanocephala & Busquereta de cap negre & Curruca cabecinegra \\
\hline Sylvia hortensis & Busquereta emmascarada & Curruca mirlona \\
\hline Sylvia communis & Busquereta vulgar & Curruca zarcera \\
\hline Sylvia atricapilla & Busquereta de capell & Curruca capirotada \\
\hline Phylloscopus bonelli & Mosquiter pàl.lid & Mosquitero papialbo \\
\hline Phylloscopus sibilatrix & Mosquiter xiulaire & Mosquitero silbador \\
\hline Phylloscopus collybita & Mosquiter comú & Mosquitero común \\
\hline Phylloscopus trochilus & Mosquiter de passa & Mosquitero musical \\
\hline Regulus regulus & Reiet coronat & Reyezuelo sencillo \\
\hline Regulus ignicapilla & Reiet safraner & Reyezuelo listado \\
\hline \multicolumn{3}{|l|}{ Fam. Muscicapidae } \\
\hline Muscicapa striata & Mastegatatxes & Papamoscas gris \\
\hline Ficedula hypoleuca & Papamosques blanquet & Papamoscas cerrojillo \\
\hline \multicolumn{3}{|l|}{ Fam. Aegithalidae } \\
\hline Aegithalos caudatus & Senyoreta & Mito común \\
\hline \multicolumn{3}{|l|}{ Fam. Paridae } \\
\hline Lophophanes cristatus & Capellanet & Herrerillo capuchino \\
\hline Periparus ater & Ferreret negre & Carbonero garrapinos \\
\hline Cyanistes caeruleus & Ferreret blau & Herrerillo común \\
\hline Parus major & Totestiu & Carbonero común \\
\hline \multicolumn{3}{|l|}{ Fam. Tichodromadidae } \\
\hline Tichodroma muraria & Pela-roques & Treparriscos \\
\hline \multicolumn{3}{|l|}{ Fam. Certhidae } \\
\hline Certhia brachydactyla & Roda-soques comú & Agateador europeo \\
\hline \multicolumn{3}{|l|}{ Fam. Oriolidae } \\
\hline Oriolus oriolus & Bacorer & Oropéndola europea \\
\hline
\end{tabular}




\begin{tabular}{|c|c|c|}
\hline Fam. Laniidae & & \\
\hline Lanius meridionalis & Botxí & Alcaudón real \\
\hline Lanius senator & Capsot & Alcaudón común \\
\hline \multicolumn{3}{|l|}{ Fam. Corvidae } \\
\hline Garrulus glandarius & Gaio & Arrendajo euroasiático \\
\hline Pica pica & Blanca & Urraca común \\
\hline Pyrrhocorax pyrrhocorax & Gralla de bec roig & Chova piquirroja \\
\hline Corvus corone & Cucala & Corneja negra \\
\hline Corvus corax & Corb & Cuervo grande \\
\hline \multicolumn{3}{|l|}{ Fam. Sturnidae } \\
\hline Sturnus vulgaris & Estornell vulgar & Estornino pinto \\
\hline Sturnus unicolor & Estornell negre & Estornino negro \\
\hline \multicolumn{3}{|l|}{ Fam. Passeridae } \\
\hline Passer domesticus & Teulat comú & Gorrión común \\
\hline Passer montanus & Teulat castellà & Gorrión molinero \\
\hline Petronia petronia & Teulat de serra & Gorrión chillón \\
\hline \multicolumn{3}{|l|}{ Fam. Fringillidae } \\
\hline Fringilla coelebs & Pinsà comú & Pinzón vulgar \\
\hline Fringilla montifringilla & Pinsà mec & Pinzón real \\
\hline Serinus serinus & Gafarró & Serín verdecillo \\
\hline Carduelis citrinella & Verderolet & Verderón serrano \\
\hline Chloris chloris & Verderol & Verderón común \\
\hline Carduelis carduelis & Cagarnera & Jilguero europeo \\
\hline Spinus spinus & Gavatxet & Jilguero lúgano \\
\hline Linaria cannabina & Passerell & Pardillo común \\
\hline Loxia curvirostra & Trencapinyes & Piquituerto común \\
\hline Coccothraustes coccothraustes & Trencapinyols & Picogordo común \\
\hline \multicolumn{3}{|l|}{ Fam. Embericidae } \\
\hline Emberiza citrinella & Verderola & Escribano cerillo \\
\hline Emberiza cirlus & Sit groc & Escribano soteño \\
\hline Emberiza cia & Sit de serra & Escribano montesino \\
\hline Emberiza hortulana & Hortolà & Escribano hortelano \\
\hline Emberiza calandra & Cruixidell & Escribano triguero \\
\hline
\end{tabular}


Tabla 2: Relación de especies de mamíferos registrados en la zona de estudio.

\begin{tabular}{|c|c|c|}
\hline NOMBRE CIENTÍFICO & NOMBRE VALENCIANO & NOMBRE CASTELLANO \\
\hline \multicolumn{3}{|l|}{ Fam. Canidae } \\
\hline Vulpes vulpes & Rabosa & Zorro rojo \\
\hline \multicolumn{3}{|l|}{ Fam. Mustelidae } \\
\hline Mustela nivalis & Mostela & Comadreja \\
\hline Martes foina & Fagina & Garduña \\
\hline Meles meles & Teixó & Tejón \\
\hline \multicolumn{3}{|l|}{ Fam. Viverridae } \\
\hline Genetta genetta & Geneta & Gineta \\
\hline \multicolumn{3}{|l|}{ Fam. Felidae } \\
\hline Felis silvestris & Gat salvatge & Gato montés \\
\hline \multicolumn{3}{|l|}{ Fam. Erinaceidae } \\
\hline Erinaceus europaeus & Eriçó comú & Erizo europeo \\
\hline \multicolumn{3}{|l|}{ Fam. Soricidae } \\
\hline Crocidura russula & Musaranya comuna & Musaraña gris \\
\hline Suncus etruscus & Musaranya nana & Musarañita \\
\hline \multicolumn{3}{|l|}{ Fam. Leporidae } \\
\hline Lepus granatensis & Llebre & Liebre ibérica \\
\hline Oryctolagus cuniculus & Conill & Conejo \\
\hline \multicolumn{3}{|l|}{ Fam. Suidae } \\
\hline Sus scrofa & Porc senglar & Jabalí \\
\hline \multicolumn{3}{|l|}{ Fam. Cervidae } \\
\hline Capreolus capreolus & Cabirol & Corzo \\
\hline \multicolumn{3}{|l|}{ Fam. Bovidae } \\
\hline Capra pyrenaica & Cabra salvatge & Cabra montés \\
\hline \multicolumn{3}{|l|}{ Fam. Rhinolophidae } \\
\hline Rhinolophus hipposideros & Rata de ferradura menuda & Murciélago pequeño de herradura \\
\hline \multicolumn{3}{|l|}{ Fam. Vespertilionidae } \\
\hline Myotis escalerai & Rata penada ibèrica & Murciélago ratonero ibérico \\
\hline Pipistrellus pipistrellus & Rata penada comuna & Murciélago enano \\
\hline Pipistrellus pygmaeus & Rata penada de Cabrera & Murciélago de Cabrera \\
\hline Pipistrellus kublii & Rata penada de vores clares & Murciélago de borde claro \\
\hline Hypsugo savii & Rata penada muntanyenca & Murciélago montañero \\
\hline Eptesicus serotinus/isabellinus & Rata penada d'horta & Murciélago hortelano \\
\hline
\end{tabular}




\begin{tabular}{c|c|c}
\hline Barbastella barbastellus & Rata penada de bosc & Barbastela \\
\hline Fam. Molossidae & & Murciélago rabudo \\
\hline Tadarida teniotis & Rata penada de cua llarga & Ardilla roja \\
\hline Fam. Sciuridae & & \\
\hline Sciurus vulgaris & Esquirol & Rata de agua \\
\hline Fam. Cricetidae & & Topillo de Cabrera \\
\hline Arvicola sapidus & Talpó d'aigua & \\
\hline Microtus cabrerae & Talpó de Cabrera & Ratón de campo \\
\hline Fam. Muridae & & Ratón casero \\
\hline Apodemus sylvaticus & Ratolí de bosc & Ratón moruno \\
\hline Mus musculus & Ratolí comú & Lirón careto \\
\hline Mus spretus & Ratolí mediterrani & \\
\hline Eliomys quercinus & Rata cellarda & \\
\hline
\end{tabular}

\section{REFERENCES}

De Jaime, C. \& Herrero, F. (2007). El chopo cabecero en el sur de Aragón. La identidad de un paisaje. Colección El Patrimonio Olvidado, no 3. Centro de Estudios del Jiloca. Calamocha (Teruel). 193 pp.

Gutiérrez, J., Sabater, M., Velázquez, J., Crespo, J., Cervera, F., Vilalta, M.\& Monsalve, M.A. (2021). Nuevas citas de Barbastella barbastellus en la Comunidad Valenciana. VIII Jornadas SECEMU. Murcia.

López, G. (2001). Los árboles y arbustos de la Peninsula Ibérica e Islas Baleares. Tomo I. Mundi-Prensa. Madrid. $861 \mathrm{pp}$.
Lorente, L. (2014). Los murciélagos de las formaciones de chopo cabecero del río Pancrudo (Comarca del Jiloca). Centro de Estudios del Jiloca. Informe inédito.

Mateo, G., Crespo, M. B. \& Laguna, E. (2011). Flora Valentina. Volumen I. Fundación de la Comunidad Valenciana para el Medio Ambiente. Valencia. 540 pp.

Méndez, M. (2009). Los insectos saproxílicos en la Península Ibérica: Qué sabemos y qué nos gustaría saber. Boletín Sociedad Entomológica Aragonesa, no 44: 505-512. 